\title{
Recomendação Colaborativa de Conteúdos Educacionais para Dispositivos Portáteis
}

\author{
Gustavo Reis ${ }^{1}$, Eduardo Barrére ${ }^{2}$ \\ ${ }^{1}$ Mestrando do Programa de Pós-Graduação em Ciência da Computação - Universidade \\ Federal de Juiz de Fora (UFJF). \\ ${ }^{2}$ Departamento de Ciência da Computação - Universidade Federal de Juiz de Fora
} (UFJF)

\{gustavo.reis, eduardo.barrere\}@ice.ufjf.br

\begin{abstract}
It is increasingly common to use mobile computers, especially laptops and smartphones, in educational institutions. This scenario enables new forms of communication between teachers and students, for example, the recommendation of educational content and collaboration. This paper presents an architecture that allows teachers to target content to specific groups (pre-configured) of students, according to the dynamics of access, automate content recommendation, according to the characteristics of the user's profile and other context information.
\end{abstract}

Resumo. É cada vez mais comum o uso de computadores móveis, principalmente notebooks e smartphones, em instituições de ensino. Este cenário possibilita novas formas de comunicação entre professores e alunos como, por exemplo, a recomendação de conteúdos educacionais e colaboração. Este trabalho apresenta uma arquitetura que permite ao professor direcionar conteúdos para grupos específicos (pré-configurados) de alunos e, de acordo com a dinâmica de acesso, automatizar a recomendação de conteúdos, conforme características do perfil do usuário e demais informações de contexto.

\section{Introdução}

No final de 2012, o Brasil fechou o ano com 261,81 milhões de acessos do Serviço Móvel Pessoal, registrando um crescimento de 8,08\% em relação ao ano de 2011 [Anatel 2013]. O crescimento da demanda por dispositivos móveis avançados, chamados smartphones, com poderosos processadores, maior capacidade de memória, telas maiores e sistemas operacionais abertos, ultrapassou o restante do mercado de telefonia móvel [Shin et al 2011]. Algumas funcionalidades ofertadas por esses equipamentos, como captura e exibição de vídeos, envio ou recebimento de mensagens, acesso a Internet e jogos tornaram o seu uso cada vez mais cotidiano para os usuários [Mateus and Brito 2011].

Segundo Mateus and Brito (2011), pesquisas feitas com professores revelam que poucos dizem utilizar o celular com finalidade pedagógica, outra parcela sente dificuldade no seu uso em sala de aula. Williams and Pence (2011) estudaram os resultados do uso de notebooks em sala de aula e chegaram aos seguintes resultados: 
maior aprendizado, maior autonomia (aprendizado individualizado), maior facilidade na realização de pesquisas, maior investigação empírica e aprendizado mais aprofundado.

Muitas soluções para a educação, utilizando dispositivos móveis, estão voltadas para uma área específica do conhecimento ou utilizam recursos comuns de software/hardware disponíveis no dispositivo móvel (ex. leitor de PDFs, SMS, câmera). Este trabalho apresenta uma arquitetura para recomendação de conteúdos educacionais genéricos, oriundos de diversas fontes e/ou de diferentes mídias (vídeos, áudios, imagens, textos e aplicativos).

A arquitetura proposta permite que diversos conteúdos educacionais sejam recomendados aos alunos, que devem ser inicialmente categorizados, pelo professor, em relação ao nível de conhecimento sobre um determinado assunto (como conhecimento básico, médio ou avançado). A recomendação inicial ocorre conforme indicação do professor de qual conteúdo é sugerido para cada nível de conhecimento. Novos conteúdos poderão ser indicados pelo professor ou pelos alunos durante o andamento das atividades. À medida que os alunos acessam esses conteúdos, o mecanismo de recomendação levará em consideração um conjunto de informações como perfil do usuário, informações de contexto e qualidade de experiência (QoE), para compor uma recomendação adaptada. Segundo Patrick et al (2004), QoE pode ser definida como as características das sensações, percepções e opiniões das pessoas em como elas interagem com seu meio.

A arquitetura permite também que os alunos trabalhem de forma colaborativa, onde os mesmos possam indicar conteúdos. Segundo Oliveira (2011), o trabalho colaborativo consolida a aprendizagem. Já Brito et al (2011) afirma que em um ambiente de aprendizagem colaborativa os participantes podem interagir entre eles para trocar experiências, conhecimentos, aprenderem uns com os outros e as tecnologias voltadas para a educação devem ser utilizadas como suporte ao ensino e não como uma solução para o sucesso do aprendizado. Oliveira (2011) afirma que as intervenções dos docentes foram fundamentais no uso do fórum em ambientes virtuais de aprendizado, onde foi possível transformar este recurso em um ambiente verdadeiramente colaborativo.

Para validar parte da arquitetura, foi desenvolvido o protótipo Context Aware Learning (CA-Learning) que faz uso de uma técnica de recomendação, para disponibilização de conteúdos personalizados, de acordo com o nível de conhecimento e contexto em que o usuário está inserido.

O trabalho está organizado de forma a apresentar na seção 2 os trabalhos relacionados e como esses contribuem para a arquitetura proposta. Na seção 3 é apresentada a arquitetura e suas características. A seção 4 descreve o papel da recomendação de conteúdos utilizada no CA-Learning. A seção seguinte apresenta a avaliação de uma aplicação teste desenvolvida. Por último, na seção 6, são apresentadas as conclusões e trabalhos futuros. 


\section{Trabalhos Relacionados}

Nesta seção são apresentadas algumas soluções para disponibilização de conteúdos educacionais via dispositivos portáteis. A primeira solução é conhecida como EduConnect [Luz and Fonseca 2013] e tem como proposta o apoio a aprendizagem colaborativa, fazendo uso das redes Wifi Direct para facilitar a transferência de arquivos entre alunos e professores. Um dos métodos utilizados na aprendizagem colaborativa é a investigação em grupo, no qual os estudantes trabalham em pequenos grupos para examinar, experimentar e/ou compreender temas centrais de estudo.

Em outro trabalho, o smartphone pode ser utilizado em um curso de enfermagem para reforçar o aprendizado do aluno, em qualquer momento ou local [Phillippi and Wyatt 2011]. As funções comuns do aparelho podem ser utilizadas para visualização de vídeos instrucionais, contactar o professor através do envio de mensagens, referenciar materiais, expandindo desta forma suas funcionalidades.

Já o sistema chamado mobUS [d'Oliveira and Costa 2012] tem como objetivo tornar o uso de tablets, smartphones e pda's em sala de aula benéfico através da computação sensível ao contexto. Durante uma aula de matemática elementar, funções básicas são exibidas ao aluno no dispositivo móvel, como também eventuais exercícios sendo que o resultado desses são enviados diretamente para o dispositivo do professor que analisará o desempenho da turma e quais as dificuldades individuais dos alunos.

Natalli and Menezes (2012) propuseram a construção de um framework, chamado FrameColab, para modelagem e desenvolvimento de ambientes colaborativos apoiado nos conceitos de Unidade de Produção Intelectual (UPI) e nos Veículos de Comunicação (Vcom). Segundo o autor, a proposta vai de encontro aos ambientes convencionais de colaboração que são concebidos para propósitos específicos e restritos.

O jogo chamado Sherlock Dengue, desenvolvido por Buchinger et al (2012), tem como principal motivação a colaboração distribuída na tentativa de aumentar a interação dos usuários com o jogo e o conteúdo sobre o combate contra a dengue. Segundo o autor, a colaboração realça o trabalho conjunto e as interações sociais entre os usuários.

A Tabela 1 apresenta um comparativo entre os trabalhos relacionados e a arquitetura proposta neste artigo. Foram tomados como parâmetros os itens mais citados em m-learning pelos autores Huang et al (2012), Ozdamli and Cavus (2011), Lichtnow et al (2006), Belém et al (2012) e Lemos et al (2012), como Colaboração/Interatividade (Col/Int) entre aluno-aluno e aluno-professor, Adaptação de conteúdos (Adap), Avaliação/Feedback (Aval/Feed), Recomendação de conteúdos (RecCont) e Informações de Contexto (Contexto). Outros parâmetros foram adicionados para caracterização dos trabalhos, pois são considerados relevantes na arquitetura proposta. São eles: Aplicação Específica (AppEsp) que depende da instalação de uma nova aplicação, Uso de Aplicações Residentes (AppRes) que necessita de aplicações previamente instaladas (leitor de PDF, SMS, etc) e Abordagem (se o propósito é específico para uma área ou de uso geral). 
Tabela 1. Quadro Comparativo entre os Trabalhos Relacionados e a CA-Learning

\begin{tabular}{|l|l|l|l|l|l|l|l|l|}
\hline & AppEsp & AppRes & Col/Int & RecCont & Aval/Feed & Contexto & Abordagem & Adap \\
\hline EduConnect & Sim & Sim & Sim & Não & Não & Não & Geral & Não \\
\hline $\begin{array}{l}\text { Smartphone } \\
\text { Nurse Educ }\end{array}$ & Não & Sim & Sim & Não & Não & Não & Enfermagem & Não \\
\hline mobUS & Sim & Não & Não & Sim & Sim & Sim & Matemática & Não \\
\hline S. Dengue & Sim & ------- & Sim & Não & Sim & Não & Específica & ---- \\
\hline FrameColab & ------- & ------- & Sim & Não & Não & Não & Geral & Não \\
\hline CA-Learning & Sim & Sim & Sim & Sim & Sim & Sim & Geral & Sim \\
\hline
\end{tabular}

Pelo comparativo apresentado na Tabela 1 é possível visualizar que as soluções apresentadas pelos autores não utilizam a maioria dos conceitos mais comuns do $\mathrm{m}$ learning. A arquitetura proposta procura fazer uso destes conceitos para um melhor resultado na recomendação e adaptação de conteúdos educacionais e aplicação. Um ponto negativo da arquitetura proposta é que a mesma depende de outras aplicações préexistentes no dispositivo, como leitor de PDF e reprodutor de vídeo. Esta situação limita atividades como dimensionar o quanto o aluno manipulou cada mídia. Fato esse que pode ser importante numa abordagem relacionada ao consumo real da mídia.

\section{Arquitetura do Sistema}

Segundo Ozdamli and Cavus (2011), o m-learning deve promover a interação entre aluno/aluno e aluno/professor, além da variação de conteúdo de acordo com a necessidade pedagógica do aluno. É preciso criar mecanismos para permitir interatividade, colaboração entre grupos e feedback dos alunos. De acordo com Wu et al (2012), um ambiente de aprendizagem deve ser adaptativo, como tipos de dispositivos certamente têm impacto no m-learning. A arquitetura proposta, vide Figura 1, leva em consideração estas questões.

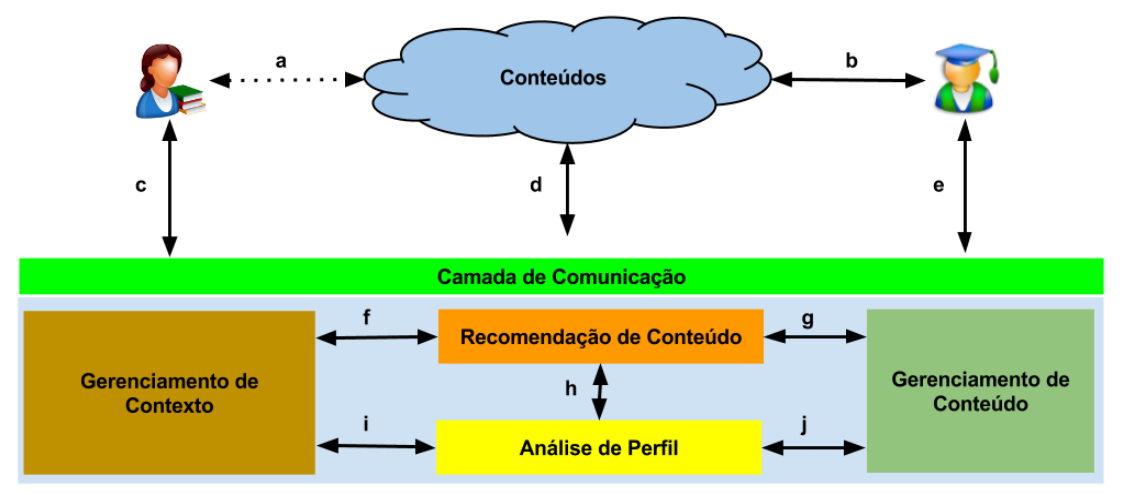

Figura 1. Arquitetura do sistema proposto

Inicialmente, o professor definirá os critérios (fluxo e) através dos quais os conteúdos educacionais serão inicialmente classificados, definindo assim uma categorização do perfil inicial para os alunos de uma turma. Em seguida, o professor fará uma associação dos conteúdos (fluxos b, e) com os critérios definidos, conforme classificação inicial informada pelo mesmo, ao módulo Gerenciamento de Conteúdo. Esta recomendação de conteúdo indicado para um determinado perfil de aluno da turma 
será a recomendação inicial. Todos os conteúdos classificados para aquele perfil são potencialmente recomendáveis para os alunos desse mesmo perfil.

No primeiro acesso do aluno na aplicação mobile, fluxo (c), o módulo Análise de Perfil, após identificação do aluno, avaliará o nível de conhecimento deste aluno em um determinado assunto, conforme critérios estabelecidos inicialmente pelo professor. Esta análise poderá ser feita através de informações prévias (via Ambiente Virtual de Aprendizagem ou outra fonte de informações acadêmicas do aluno) ou informações disponibilizadas pelo próprio aluno através de um formulário. São enviadas também, fluxo (c), informações de contexto sobre características do dispositivo (tamanho de tela, resolução, se está em movimento, etc.) para que o módulo Gerenciamento de Contexto realize, se necessário, sugestões de adaptações de conteúdos, como recomendar conteúdos que tenham áudio caso o aluno esteja em movimento ou adaptar a aplicação dependendo da luminosidade externa. Segundo Hansen (2012), aplicações com comportamento adaptativo, aumentam a previsibilidade e a capacidade em funcionar de forma eficiente sob uma ampla gama de condições.

O próximo passo é a Recomendação de Conteúdo propriamente dita. Este módulo é responsável por coletar as informações de contexto (f), perfil (h), conteúdos sugeridos ( $\mathrm{g}$, d) e através de uma técnica de recomendação, analisar estes dados recuperados e fazer a recomendação de conteúdos para o aluno, levando em consideração seu perfil inicial, conteúdos que ele acessou, suas informações de contexto e os conteúdos acessados por colegas que tenham um perfil similar ao seu.

À medida que o aluno acessa os conteúdos sugeridos, também são recomendados pequenos questionários, no estilo Quiz, que permitem atualizar o perfil do aluno conforme seu desempenho. Esses questionários são formados por perguntas de múltipla escolha e elaborados previamente pelo professor. Além disso, o próprio aluno pode pesquisar (fluxo a) e fazer sugestões (fluxo c) de conteúdos, mas o mesmo deverá passar por uma avaliação prévia do professor antes de ser disponibilizado para recomendação.

Como complementação da arquitetura, os fluxos (i) e (j) podem ser utilizados para realizar futuras atividades de mineração de dados, buscando assim informações não explicitamente apresentadas pelos módulos isoladamente. Este tipo de ação pode ser importante para busca de conhecimento sobre o perfil de acesso dos alunos.

\section{Recomendação de Conteúdo}

Um sistema de recomendação de conteúdo tem por objetivo analisar as preferências do usuário e também suas necessidades, recomendando conteúdos pertinentes a estas características. Segundo Lichtnow et al (2006), os sistemas de recomendações atuais são implementados mediante o uso de diversas técnicas, variando em função do objetivo a ser atingido e o tipo de item a ser recomendado.

Existem várias técnicas utilizadas para recomendação de conteúdo como memory-based, model-based, Top- $N$, grafos gerados a partir das visualizações de usuários, entre outras. Estas técnicas buscam criar um conjunto ordenado de itens (rankings), com certa semelhança, para serem recomendados a um usuário. Duarte (2013) utilizou como técnica de comparação entre objetos a Similaridade de Cosseno para recomendação de vídeos. Belém et al (2012) levou em consideração não somente a 
relevância, mas adicionou também novidade e diversidade como aspectos essenciais para recomendações de tags. Foram utilizadas duas estratégias de recomendação: uma delas está baseada em relevance feedback e a outra técnica utilizada é a programação genética (PG) levando em consideração relevância, novidade e diversidade.

Lemos et al (2012) propôs a recomendação de fotos através da similaridade, sendo calculada por uma média ponderada onde os pesos são as atividades que o usuário está desenvolvendo.

Segundo Lathia et al (2008), as técnicas mais conhecidas para calcular similaridade dos usuários produzem o cálculo de similaridades que tendem a ficar próximas entre elas. Sendo assim, para a recomendação de conteúdos da arquitetura proposta, são desempenhadas as seguintes etapas:

- Como recomendação inicial, os alunos recebem conteúdos educacionais prédefinidos (template) de acordo com o seu nível de conhecimento. Este template é definido pelo professor e é analisado pelo módulo Gerenciamento de Conteúdo para ser classificado de acordo com o perfil do aluno;

- Juntamente com estes conteúdos educacionais pré-definidos, os alunos recebem outros conteúdos através do cálculo de Similaridade de Cosseno, sugeridos após a recomendação inicial, sejam eles feitos pelo professor ou por alunos. Desta forma os conteúdos adicionais não são pré-classificados pelo professor, mas são recomendados junto com o template de acordo com a similaridade entre eles;

- À medida que alunos de mesmo perfil interagem com seus conteúdos, é feito um novo cálculo, através do método Concordance-based, para recomendar conteúdos semelhantes dos conteúdos mais acessados. O método Concordancebased baseia-se na classificação de similaridade para gerar coeficientes que visam encontrar o grau de aceitação sobre a interseção de perfis de dois usuários. Desta forma, é possível criar um ranking de conteúdos mais acessados entre os alunos de mesmo perfil e fazer a recomendação deste ranking.

Com a recomendação de conteúdos personalizados, o aluno terá à disposição conteúdos educacionais de acordo com suas características relacionadas ao seu conhecimento (indicado previamente), podendo receber e acessar conteúdos educacionais mais adequados às suas necessidades.

\section{Protótipo}

Como projeto inicial, foi desenvolvido um protótipo para validar parte da arquitetura, vide Figura 1, chamado Context Aware Learning (CA-Learning). O CA-Learning contempla as seguintes funcionalidades: identificação do aluno $(\operatorname{login})$, escolha do assunto a ser estudado, questionário para verificar nível de conhecimento do assunto escolhido, análise do perfil, recomendação de conteúdos. O CA-Learning foi desenvolvido em dois módulos: um para a plataforma Android e outro voltado para web. O módulo desenvolvido para o dispositivo móvel é responsável por identificar o aluno e seu perfil. A partir da coleta dessas informações, as mesmas são envias para o módulo $w e b$. Ao receber as informações, o módulo web analisa o perfil do aluno (através de uma média ponderada) e faz a recomendação de conteúdos de acordo com seu perfil. 


\subsection{Cenário}

Foram feitos dois tipos de levantamentos de dados. O primeiro teve como objetivo diagnosticar quais os momentos de pico de requisições de conteúdos feitos durante o momento da aula e os tipos de materiais consumidos. O segundo levantamento, objeto de estudo deste trabalho, teve como objetivo fazer o levantamento das opiniões e perfis dos alunos que participaram do experimento. Os testes foram feitos com três turmas de um Curso Técnico em Informática de uma Instituição Federal de ensino. A atividade teve duração aproximada de 50 minutos para cada turma.

Os alunos foram encaminhados para o laboratório, instruídos sobre o objetivo do trabalho e receberam treinamento no uso do aplicativo. Após o treinamento, os alunos puderam fazer uso do aplicativo e consumir os conteúdos recomendados. Foram disponibilizados 45 conteúdos que abordaram assuntos relacionados a Banco de Dados, Algoritmos, Redes de Computadores e Sistemas Operacionais. Esses conteúdos, de autoria do próprio professor ou adquiridos da web, foram classificados como básico ou avançado, sendo disponibilizados nos formatos imagem, texto e vídeo. Finalizado o experimento, os alunos receberam um questionário composto por cinco questões, todas são de múltipla escolha, sendo que as questões 3, 4 e 5 possuem um espaço para que os alunos pudessem expressar suas opiniões. Este questionário tem o objetivo de traçar os perfis e opiniões dos alunos em relação às características dos dispositivos utilizados para acesso a Internet, a relevância do uso do smartphone como recurso para o ensino.

\subsection{Análise dos dados coletados}

O questionário foi aplicado em três turmas, totalizando 50 alunos participantes do experimento. As cinco perguntas que compõem o questionário são: Qual o tipo de dispositivo você costuma utilizar para acessar a Internet? Sobre o objetivo da aplicação, qual a importância deste para o aprendizado? Fazer uso do celular como um recurso para o ensino é relevante? Os tipos de materiais disponibilizados são suficientes? Qual o tipo de material você prefere fazer uso para os estudos complementares?

Foram destacadas duas perguntas que levam em consideração a importância do uso de tecnologias voltadas para a educação e a preferência do tipo de mídia a ser consumido. O gráfico da Figura 2-a (Sobre o objetivo da aplicação, qual a importância deste para o aprendizado?) mostra que as turmas 1 e 2 tem opiniões divididas (bom e excelente) sobre a importância do uso desse tipo de aplicação. Sendo que na turma 3, $66,7 \%$ dos alunos acham bom o uso da aplicação no auxílio ao aprendizado. Conclui-se, portanto, que independente da turma, os alunos consideram esse tipo de aplicação importante para o aprendizado (quase $100 \%$ dos participantes).

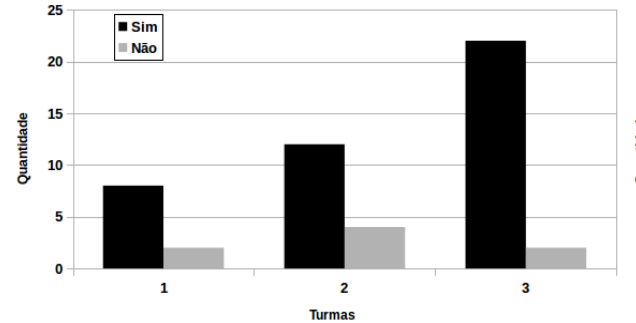

(a)

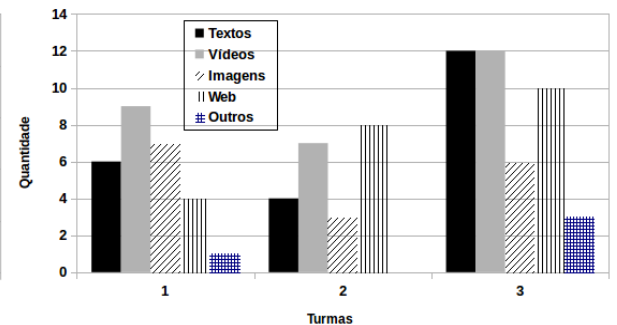

(b)

Figura 2. Gráficos apresentando resultados da pesquisa 
O gráfico da Figura 2-b (Qual o tipo de material você prefere fazer uso para os estudos complementares?) apresenta as preferências dos tipos de mídias a serem utilizadas como estudos complementares. Fazendo uma análise conjunta, os alunos demonstraram um maior interesse em fazer uso de vídeos como material complementar de estudos $(30,4 \%)$, seguido de textos $(23,91 \%)$. Nota-se ainda que existe uma preferência considerável na intenção do uso de conteúdos web, cerca de 23,91\% (em especial para as turmas 2 e 3 ).

Além disso, do total de participantes do experimento, cerca de $37,84 \%$ utilizam o smartphone para acesso a Internet e $33,78 \%$ fazem uso de notebook para o mesmo tipo de acesso. Sendo assim, pode ser levado em consideração o motivo pelo qual os alunos têm uma grande preferência no consumo de vídeos, pelo fato de usar dispositivos que possuem telas menores (como smartphone). E o interesse pelo consumo de materiais web pode ser pressuposto pela facilidade de encontrar conteúdos de interesse fazendo uso de ferramentas de buscas automáticas.

Vale ainda destacar algumas opiniões dos alunos em relação ao projeto:

- "Acesso de qualquer lugar"

- "Pois assim utilizamos o meio de comunicação que mais usamos para estudar"

- "Pois é um dispositivo onde podemos ter acesso a qualquer momento"

\section{Conclusões e Trabalhos Futuros}

A utilização de dispositivos móveis aumentou a demanda por consumo de informações, principalmente a discussão do seu uso na escola, onde o professor tenha condições de utilizar este recurso como um aliado ao ensino.

A arquitetura proposta busca reunir as principais características para um sistema de aprendizado móvel como promover a colaboração entre os alunos, fazer recomendação de conteúdos de acordo com perfil e preferências do usuário. Além disso, a arquitetura deve fazer uso de informações de contexto para adaptar conteúdos e sistema de acordo com as características do dispositivo, do ambiente externo e atividades desenvolvidas pelo aluno durante sua interação com o aplicativo.

O protótipo desenvolvido, como parte da arquitetura, buscou fazer o levantamento do perfil de cada aluno, permitindo desta forma, entregar materiais recomendados para cada nível de conhecimento do mesmo. Com a evolução no conhecimento sobre comportamento de interação desses alunos, o CA-Learning tem condições de recomendar outros conteúdos, a partir de conteúdos adicionais disponibilizados pelo professor ou pelos próprios alunos, promovendo de forma transparente e natural a colaboração entre eles.

Como trabalhos futuros a arquitetura deverá suportar:

- integração com uma plataforma de ensino, desta forma poderá calcular melhor o perfil do usuário de acordo com sua evolução durante o curso;

- avaliação mais detalhada e possíveis correções na dinâmica de formação e atualização do perfil do aluno;

- possibilitar uma melhor definição do estilo de aprendizagem de cada aluno. Segundo Huang et al (2012), o estilo de aprendizagem é o comportamento no 
qual o aluno observa e interage com o ambiente de aprendizado para obter conhecimento e habilidades;

- uso da Lógica Fuzzy para validar o nível de conhecimento e evolução dos alunos sobre os conteúdos recomendados. Martins (2013) utilizou a Lógica Fuzzy para adaptação de conteúdo de acordo com o nível de conhecimento do aluno em um curso de inglês à distância;

- apesar de Lathia et al (2008) afirmar que as técnicas mais conhecidas para calcular similaridade dos usuários produzem o cálculo de similaridades que tendem a ficar próximas entre elas, é importante realizar um estudo comparativo entre os cálculos de similaridade mais conhecidos para aprimorar a recomendação de conteúdo no cenário previsto para o CA-Learning.

\section{Referências}

Anatel (2013) "Relatório Anual Grupo de Dados da SPV 2012”, In: Superintendência de Serviços Privados, Dados, Móvel e Satélite http://www.anatel.gov.br/Portal/verificaDocumentos/documento.asp?numeroPublica $\mathrm{cao}=296023 \&$ pub $=$ principal $\&$ filtro $=1 \&$ documentoPath $=296023$. pdf.

Belém, F. M., Martins, E. F., Almeida, J. M. and Gonçalves, M. A. (2012) "Exploiting relevance, novelty and diversity in tag recommendation", In: Proceedings of the 18th Brazilian symposium on Multimedia and the web, p. 297-300. Publishing ACM.

Brito, J. A., de Melo Filho, I. J., Carvalho, R. S., de Melo, R. M. and Gomes, A. S. (2011) "Interfaces colaborativas para atividades assíncronas em fórum de discussão", In: Anais do XXII Simpósio Brasileiro de Informática na Educação, vol. 22, no. 1.

Buchinger, D., Hounsell, M. D. S. and Dias, C. (2012) "Colaboratividade em um Jogo Eletrônico para Ensino sobre Dengue”, In: Anais XXIII do Simpósio Brasileiro de Informática na Educação, vol. 23, n. 1.

d'Oliveira, R. B. and Costa, D. P. (2012) "Benefícios da Computação Pervasiva na Educação e mobUS, Um Sistema Móvel no Auxilio à Aprendizagem", In: Anais do XXIII Simpósio Brasileiro de Informática na Educação, vol. 23, n. 1.

Duarte, D., Pereira, A. and Davis, C. (2013) "Modeling, characterizing and recommendation in multimedia web content services", In: Proceedings of the 19th Brazilian symposium on Multimedia and the web, p. 265-268. Publishing ACM.

Hansen, J. (2012) “An investigation of smartphone applications: Exploring usability aspects related to wireless personal area networks, context-awareness, and remote information access", In: School of Information Systems, Computing and Mathematics. Brunel University.

Huang, H. C., Wang, T. Y. and Hsieh, F. M. (2012) "Constructing an Adaptive Mobile Learning System for the Support of Personalized Learning and Device Adaptation", In: Procedia-Social and Behavioral Sciences, vol. 64, p. 332-341.

Lathia, N., Hailes, S., and Capra, L. (2008) "The effect of correlation coefficients on communities of recommenders", In: Proceedings of the 2008 ACM symposium on Applied computing, p. 2000-2005. Publishing ACM. 
Lemos, F. D., Carmo, R. A., Viana, W. and Andrade, R. (2012) "Improving photo recommendation with context awareness", In: Proceedings of the 18th Brazilian symposium on Multimedia and the web, p. 321-330. Publishing ACM.

Lichtnow, D., Garin, R. S., Palazzo, L. A. M., Loh, S., Kampff, A. J. C., Primo, T. and Lima, J. V. D. (2006) "O uso de técnicas de recomendação em um sistema para apoio à aprendizagem colaborativa”, In: Revista Brasileira de Informática na Educação, vol. 14, n. 3 .

Luz, J. W. P., and Fonseca, L. C. C. (2013) "EduConnect: uma ferramenta de apoio à aprendizagem colaborativa para dispositivos móveis em redes MANET", In: Anais do XXIV Simpósio Brasileiro de Informática na Educação, vol. 24, n. 1.

Mateus, M. C. and Brito, G. S. (2011) "Celulares, Smartphones e Tablets na sala de aula: complicações ou contribuições?" In: X Congresso Nacional em Educação Educere, vol. 10.

Martins, Valéria Farinazzo et al (2013) "Sistema Hipermídia Adaptativa para o Ensino de Inglês” In: Anais do XXIV Simpósio Brasileiro de Informática na Educação, vol. 24, n. 1 .

Natalli, E. L., and Menezes, C. S. (2012) "FrameColab: Um framework para a criação de Ambientes Colaborativos", In: Anais do Simpósio Brasileiro de Informática na Educação, vol. 23, no. 1.

Oliveira, G. P. (2011) “O fórum em um ambiente virtual de aprendizado colaborativo", In: Revista Digital de Tecnologia Educacional e Educação a Distância, vol. 2, n. 1.

Ozdamli, F., and Cavus, N. (2011) "Basic elements and characteristics of mobile learning", In: Procedia-Social and Behavioral Sciences, vol. 28, p. 937-942.

Patrick, A. S., Singer, J., Corrie, B., Noël, S., El Khatib, K., Emond, B. and Marsh, S. (2004) "A QoE sensitive architecture for advanced collaborative environments", In: Quality of Service in Heterogeneous Wired/Wireless Networks QSHINE 2004. First International Conference on, p. 319-322, Publishing IEEE.

Phillippi, J. C. and Wyatt, T. H. (2011) "Smartphones in nursing education", In: Computers Informatics Nursing, vol. 29(8), p. 449-454.

Shin, D. H., Shin, Y. J., Choo, H. and Beom, K. (2011) "Smartphones as smart pedagogical tools: Implications for smartphones as u-learning devices", In: Computers in Human Behavior, vol . 27(6), p. 2207-2214, Publishing Elsevier.

Williams, A. J. and Pence, H. E. (2011) "Smart phones, a powerful tool in the chemistry classroom", In:Journal of Chemical Education, 88(6), p. 683-686.

Wu, W. H., Jim Wu, Y. C., Chen, C. Y., Kao, H. Y., Lin, C. H. and Huang, S. H. (2012) "Review of trends from mobile learning studies: A meta-analysis.Computers \& Education", In: Computers \& Education, vol. 59, p. 817-827, Publishing Elsevier. 